social interdependence and cohesion within the inhabitants of small towns not only contribute to a successful integration but also to the low levels of violence and crime. For this example to work elsewhere, equal working and education opportunities as well as social and cultural activeness of people should be considered.

\section{THE RELATIONSHIP BETWEEN INJURIES BY ACCIDENTS AND VIOLENCE IN CHILDREN UNDER THE ASPECT OF THE NEW "PREVENTION LAW" IN GERMANY}

Johann Böhmann, Anna Stumpe. Kinderklinik Klinikum Delmenhorst, DIG-Delmenhorster Institut Für Gesundheitsförderung, Delmenhorst Germany

\subsection{6/injuryprev-2016-042156.275}

Background Unintentional and intentional Injuries are not quite different and do not obligatory require different approaches particularly concerning their Prevention. This has to be explained and discussed under the new German "Kinderschutzgesetz" as well as the "Präventionsgesetz".

Methods Common determinants for injuries and violence are highlighted. Fatal examples are described. Consequences on the local (community) level are pointed out by analysing the different networks in 6 different communities in northern Germany, one of them is the first German "Safe community" of Delmenhorst.

Results The Differences between the two "systems": The medical system and the general community service and child protection services are fundamental. There is not only a general deficiency of information and professional routines but even more important differences in attitudes and engagement. This becomes more obvious by comparing networks in different communities. Unfortunately these differences seem to be state aided by the concurrent jurisdiction.

Conclusions Abstract The systemic problem can only be solved on a community level. Local experiences have to be expanded to a regional or national level.

\section{FOSTERING INQUIRY AND MULTI-SECTORAL HUMILITY USING THE MULTI-SECTORAL INFLUENCES MATRIX}

${ }^{1,2}$ Carolyn Cumpsty-Fowler. ' Johns Hopkins University School of Nursing; 'Johns Hopkins Bloomberg School of Public Health, USA

\subsection{6/injuryprev-2016-042156.276}

Background Daniel Boorstin believed: "The greatest obstacle to discovery is not ignorance, it is the 'illusion of knowledge". As the scope and complexity of injury and violence prevention and control challenges have grown, it has become evident that no single discipline can provide the solution. Effective public-private and non-traditional partnerships are needed both to understand and address the problem; and to remove the many obstacles posed by competing priorities and opposing interests. Each sector of a community, and even sub-groups of sectors, have their own culture, priorities and "language". Even if we know that a stakeholder group exists, we may not appreciate how much we do not know about their culture.

Objectives 1. Describe the concept of multi-sectoral humility. 2. Introduce the Multi-Sectoral Influences Matrix. 3. Illustrate the relevance of the matrix in education, research and practice.
Results The need for the Multi-Sectoral Influences Matrix was inspired by practitioners. Its design and application has been honed over 15 years with feedback from hundreds of students in various disciplines and training settings. This presentation will include examples of breakthrough insights cited by students, as well as opportunities discovered through assets-focused inquiry.

Conclusions Challenging assumptions is a key component of critical thinking. No matter where we work in the world, the focus of our injury and violence prevention and control efforts, our level of training, or perceived need for resources and collaboration, we need to develop multi-sectoral humility. Until we do, we will not recognise the full potential of multi-sectoral collaboration.

\section{INSIGHT AND TOP VIEW: ANALYSING CHILD SAFETY NETWORKS IN SIX GERMAN MUNICIPALITIES IN 2014}

${ }^{1}$ Anna Stumpe, ${ }^{1,2}$ Johann Böhmann. 'Delmenhorster Institut Für Gesundheitsförderung, Germany; ${ }^{2}$ Klinikum Delmenhorst, Germany

\subsection{6/injuryprev-2016-042156.277}

Background Networking is evidently a crucial way to promote safety in municipalities. Constructivistic theories suppose that people adjust their behaviour to their own experiences rather than to explicit rules. Within the project "Safety for children in and by day-care centres and municipalities" ("Kindersicherheit in Kita und Kommune"; KiKuK) which ran from 2013 to 2014 individual behavioural patterns as well as underlying attitudes were analysed to learn more about how safety is promoted in communities using networks and how promotion is approved within the target group. KiKuK covered 33 municipalities in northern Germany.

Methods Six German municipalities, five of them rural and one city, were analysed focusing on the implicit child safety networks. 216 stakeholders working in the field promoting child safety were interviewed to identify the structure and quality of the existing networks. In order to capture the real structure rather than the organisational chart we used an innovative systemic sample approach.

Additionally, we interviewed 105 parents and stakeholders to learn more about their individual attitudes towards different stakeholders.

Results The surveyed networks differ from the allocated organisational charts. Stakeholders and families clearly distribute their sympathy and work closer with the ones they like rather than with the ones they are supposed to work with. We found that there are subtle structures in municipalities that use specific stakeholders as pivots. We also found that families have a different idea of how stakeholders should act by means of safety promotion.

Conclusions To push safety promotion forward it is not sufficient to tell stakeholders and families what to do but it is necessary to also listen to them in order to meet their sympathies. If they may do what they enjoy and with whom it suits them, they will be more likely to do it. We suggest that networking should take the individual stakeholder more into account and be less of a top down process. 